\title{
Proton beam therapy to bridge or downstage locally advanced hepatocellular carcinoma to living donor liver transplantation
}

\author{
Chao-Long Chen ${ }^{1}$, Aldwin D. Ong ${ }^{1}$, Jen-Yu Cheng ${ }^{2}$, Chee-Chien Yong ${ }^{1}$, Chih-Che Lin ${ }^{1}$, Chih-Yi Chen ${ }^{1}$, \\ Yu-Fan Cheng ${ }^{3}$ \\ ${ }^{1}$ Liver Transplantation Center, Department of Surgery, Kaohsiung Chang Gung Memorial Hospital, Chang Gung University College of Medicine, \\ Kaohsiung, Taiwan, China; ${ }^{2}$ Liver Transplantation Center, Department of Radiation Oncology, Kaohsiung Chang Gung Memorial Hospital, Chang \\ Gung University College of Medicine, Kaohsiung, Taiwan, China; ${ }^{3}$ Liver Transplantation Center, Department of Diagnostic Radiology, Kaohsiung \\ Chang Gung Memorial Hospital, Chang Gung University College of Medicine, Kaohsiung, Taiwan, China \\ Correspondence to: Chao-Long Chen, MD, PhD (Hon). Liver Transplantation Center, Department of Surgery, Kaohsiung Chang Gung Memorial \\ Hospital, 123 Ta-Pei Road, Niao-Sung, Kaohsiung, Taiwan, China. Email: clchen@cgmh.org.tw.
}

Submitted Sep 14, 2021. Accepted for publication Nov 16, 2021.

doi: $10.21037 / \mathrm{hbsn}-21-379$

View this article at: https://dx.doi.org/10.21037/hbsn-21-379

\section{Introduction}

Within the last decade, progress in transplant and surgical oncology has been tremendous. In liver oncology alone, there has been concurrent technological innovation across the different adjunct fields and tiers of oncology related to hepatocellular carcinoma (HCC), progressing in quite an unprecedented rate. Significant improvements in our capacity to diagnose and prognosticate HCC with increasing sensitivity, specificity, and predictability is being witnessed. We now better understand that staging, treatment and prognostication cannot be determined on a static basis of tumor burden (i.e., tumor size and number) alone and a "one size fits all" management. Careful individualized assessment is now mandatory in consideration of intraand intertumoral heterogeneity and tumor biology in order to more precisely guide diagnostics and treatment options (1). While liver transplantation (LT) remains the most effective curative treatment given that it eradicates existing tumors and the risk for new tumors, as well as the risk for liver failure in cirrhotics, proceeding to LT ideally requires patients to fit nationally or institutionally applicable and appropriate criteria that takes into consideration not only tumor burden, but also tumor biology; living donor LT (LDLT) has the advantage of extending the option to transplant out-of-criteria patients using more precise and patient-centered survival calculators such as the Metroticket 2.0 model by Mazzaferro et al. (2). Multimodal sequential management involving a multidisciplinary team of informed liver oncology specialists is critical in decision-making particularly for locally advanced HCC (LAHCC) where bridging and downstaging therapies for potential curative final intent are increasingly becoming available viable options (1).

Radiation oncology is rapidly becoming an important modality for locoregional therapy of LAHCC, particularly where needle- or catheter-directed interventional approaches and systemic options are contraindicated or highly risky. In the recent years, radiation therapy (RT) in the form of stereotactic body radiation therapy (SBRT) has been shown to have high local control and response rates in LAHCC, especially in cases with portal vein invasion (PVI) or portal vein tumor thrombosis (PVTT); many centers have incorporated SBRT as an important complementary consideration for bridging or downstaging out-of-criteria HCC to transplantation (3). Proton beam therapy (PBT), is one of the more recent advanced forms of radiation therapy that extends management options for patients with LAHCC.

Kaohsiung Chang Gung Memorial Hospital (KCGMH) is one of the world's leaders in LDLT, with one of the highest 3-year overall survival rates at $92 \%$ (4). Since the inception of the KCGMH Proton Center for clinical use in 2018, the cyclotron (Sumitomo Heavy Industries) and three treatment rooms (all with pencil beam scanning system) have been used for PBT of over 700 cancer patients, about $20 \%$ of which were for HCC. Given the limited available published literature on PBT for HCC, we have thus far 
only considered its use for downstaging of LAHCC in patients with no other suitable locoregional alternatives, as well as for bridging therapy before LDLT in order to further reduce the risk of post-transplant recurrence.

We herein present two cases of LAHCC who were preoperatively treated with $\mathrm{PBT}$, the first as a bridge to LDLT, while the second as an attempt to downstage the patient to fit the University of California San Francisco (UCSF) criteria as mandated by the Taiwan citizens' health insurance policy, or in consideration of an elective LDLT.

\section{Kaohsiung Chang Gung Memorial Hospital proton beam therapy protocol}

\section{Planning and preparation}

The patient is placed on supine position with a vacuum bag and knee supports with arms overhead. Abdominal compression pneumatic compression belt is used for respiratory control, permitting motion up to $1 \mathrm{~cm}$. Fourdimensional computed tomography (4DCT) images are taken at $1.25 \mathrm{~mm}$ intervals. The gross tumor volume (GTV) is outlined on an average intensity projections (AIP) set of the non-enhanced 4DCT using reference contrast-enhanced magnetic resonance (MR) images; the clinical target volume (CTV) is defined by the GTV, with a $0-5 \mathrm{~mm}$ margin by clinical estimation. Another $5 \mathrm{~mm}$ is added as margin to create an internal tumor volume (ITV) to compensate for respiratory motion. Further beam direction-specific margins are then generated based on a $3.5 \%$ range of uncertainty, and a $3 \mathrm{~mm}$ setup uncertainty to create a proton planning target volume (PPTV) for pencil beam scanning proton treatment planning (single-field optimization technique) using RayStation treatment planning system (RaySearch Laboratories, AB, Stockholm, Sweden).

\section{Proton irradiation}

Our institutional protocol was adapted from the Japanese protocol $(5,6)$. Depending on the tumor location, $72.6 \mathrm{~Gy}$ [relative biological effectiveness (RBE)] in 22 fractions or 66 Gy (RBE) in 10 fractions will be prescribed. The 66 Gy (RBE) protocol is preferred if the gastrointestinal tract is not within $2 \mathrm{~cm}$ of the treatment field. The dose to the bowel loop is constrained to $50 \%$ and $65 \%$ of the prescribed dose for the 66 and 72.6 Gy protocols respectively. Daily cone-beam CT is performed during the treatment course to verify treatment positions.

\section{Case presentation}

\section{Case 1}

The first case was a 60-year-old gentleman with an Eastern Cooperative Oncology Group (ECOG) score of 0 who had a history of hepatitis $\mathrm{C}$ virus (HCV) liver cirrhosis and recurrent HCC, status post partial right hepatectomy with cholecystectomy eight years prior, and radiofrequency ablation (RFA) to a recurrent HCC in the left lateral segment (LLS) two years prior to referral. On initial work-up, the patient presented with a model for end-stage liver disease (MELD) of 14, Child-Turcotte-Pugh (CTP) B7 (bilirubin $5.98 \mathrm{mg} / \mathrm{dL}$ ) with alpha-fetoprotein (AFP) $24 \mathrm{ng} / \mathrm{mL}$ and CA19-9 $491 \mathrm{U} / \mathrm{mL}$. Magnetic resonance imaging (MRI)-magnetic resonance cholangiopancreatography (MRCP) with gadoxetate disodium contrast (Gd-EOB-DTPA) scan revealed a $3.1 \mathrm{~cm}$ segment 8 (S8) HCC that was contiguous with the middle hepatic vein (MHV) and compressing against the S8 intrahepatic ducts (IHD) resulting in upstream dilatation (Figure 1). Positron emission tomography (PET)computerized tomography (CT) scan done for this recurrent S8 HCC revealed an increased fluorodeoxyglucose (FDG) uptake with maximum standard unit value (SUVmax) of 7.7 , and tumor to non-tumor liver uptake ratio (TNR) of 2.4 (Figure 2). The decision after multidisciplinary team (MDT) discussion was to do bridging therapy with PBT prior to LDLT with the hope of improving preoperative tumor characteristics and hence also recurrence-free survival. PBT was therefore delivered to the HCC at a dose of 66 Gy (RBE) in 10 fractions (Figure 3). Repeat PET scan two weeks after completion of PBT showed good response with decreased uptake to SUVmax 4.3 and TNR 1.3 (Figure 2C,2D). Repeat liver MRI-MRCP done four weeks later showed a residual tumor size of $1.8 \mathrm{~cm}$ with decreased signal, and significantly improved S8 IHDs with a filling defect in the distal common bile duct (dCBD) (Figure 1C); AFP decreased to $6.8 \mathrm{ng} / \mathrm{mL}$ and CA199 decreased to $13.5 \mathrm{U} / \mathrm{mL}$. The patient subsequently underwent LDLT with a right hepatic graft without the MHV from his donor son. The final explant histopathology revealed a poorly differentiated S8 HCC with at least 50\% necrosis (Figure 4); final diameter was $1.5 \mathrm{~cm}$ with note of bile duct invasion; histopathology showed an infiltrative scirrhous pattern while the $\mathrm{dCBD}$ tissue removed was identified as completely necrotic HCC. The patient has since been maintained on dual-drug immunosuppression that included a calcineurin inhibitor (CNI) and a mammalian target of rapamycin (mTor) inhibitor, 

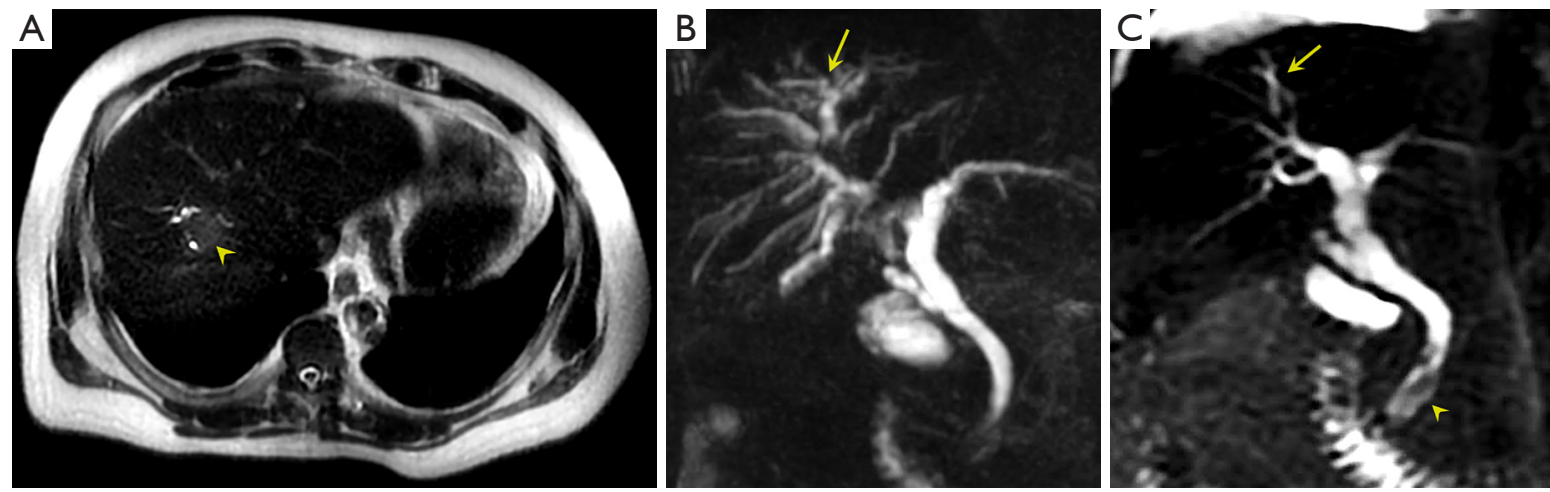

Figure 1 Magnetic resonance imaging (MRI)-magnetic resonance cholangiopancreatography (MRCP) (Case 1) showing hepatocellular carcinoma (HCC) over S8 of the liver (arrowhead) (A) with regional intrahepatic duct (IHD) dilatation (arrow) before proton beam therapy (PBT) (B). Repeat MRCP four weeks after completion of PBT shows marked decrease in IHD caliber (arrow) and identification of a filling defect at the distal common bile duct (arrowhead) (C) that was later identified as completely necrotic HCC on histopathology.
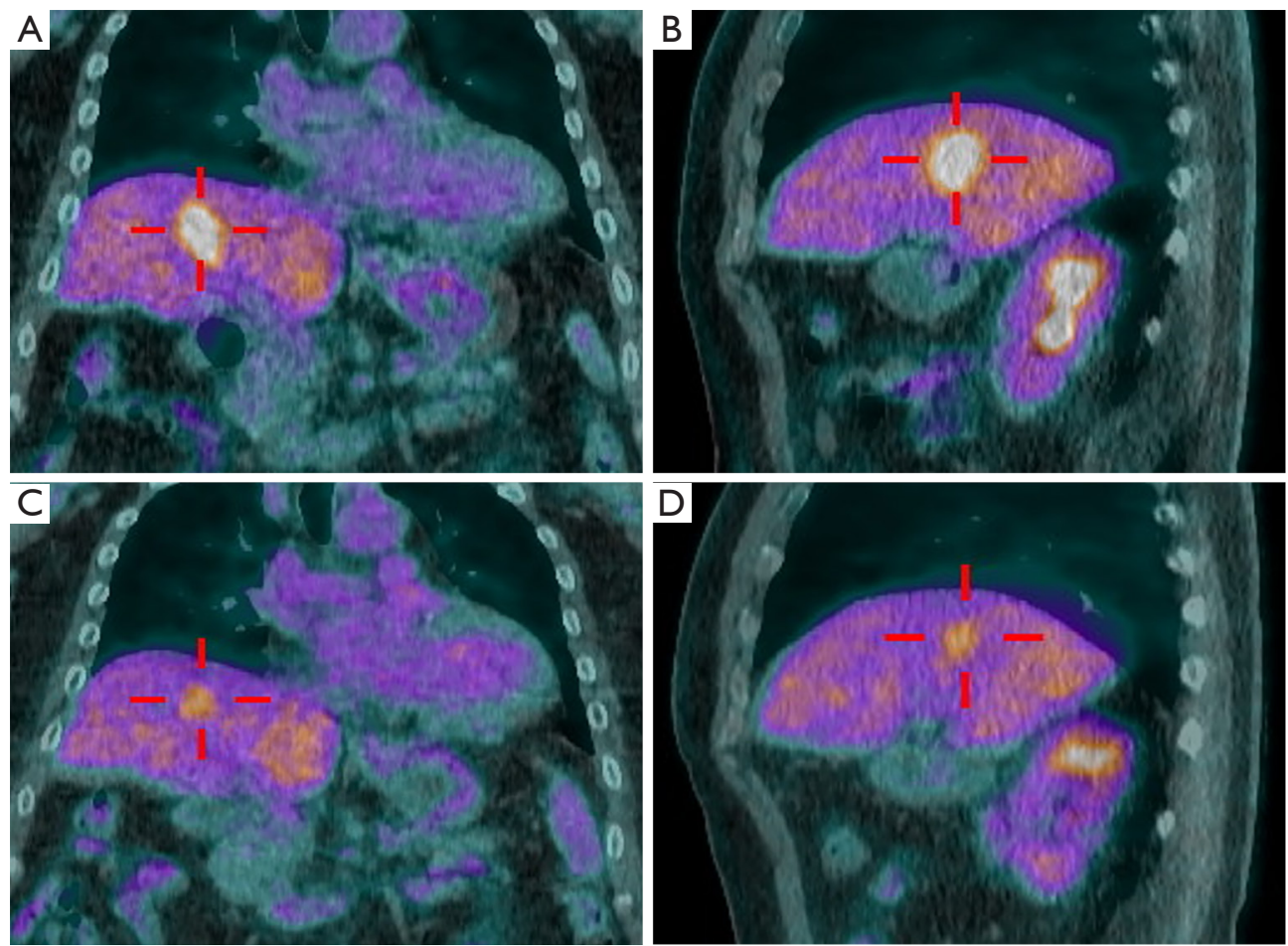

Figure 2 Positron emission tomography (PET) scan (Case 1). Coronal (A) and sagittal (B) views before proton beam therapy (PBT) showing high FDG uptake of the S8 lesion with maximum SUVmax of 7.7, and TNR of 2.4. Coronal (C) and sagittal (D) views 2 weeks after PBT showing decreased FDG uptake to SUVmax of 4.3, and TNR of 1.3 .

concurrently taken with a tyrosine kinase inhibitor (TKI) lenvatinib, and has since been without recurrent disease after 18 months of transplantation.

\section{Case 2}

The second case was a 59-year-old lady, ECOG 1, known case of $\mathrm{HCV}$ who had previously been treated 

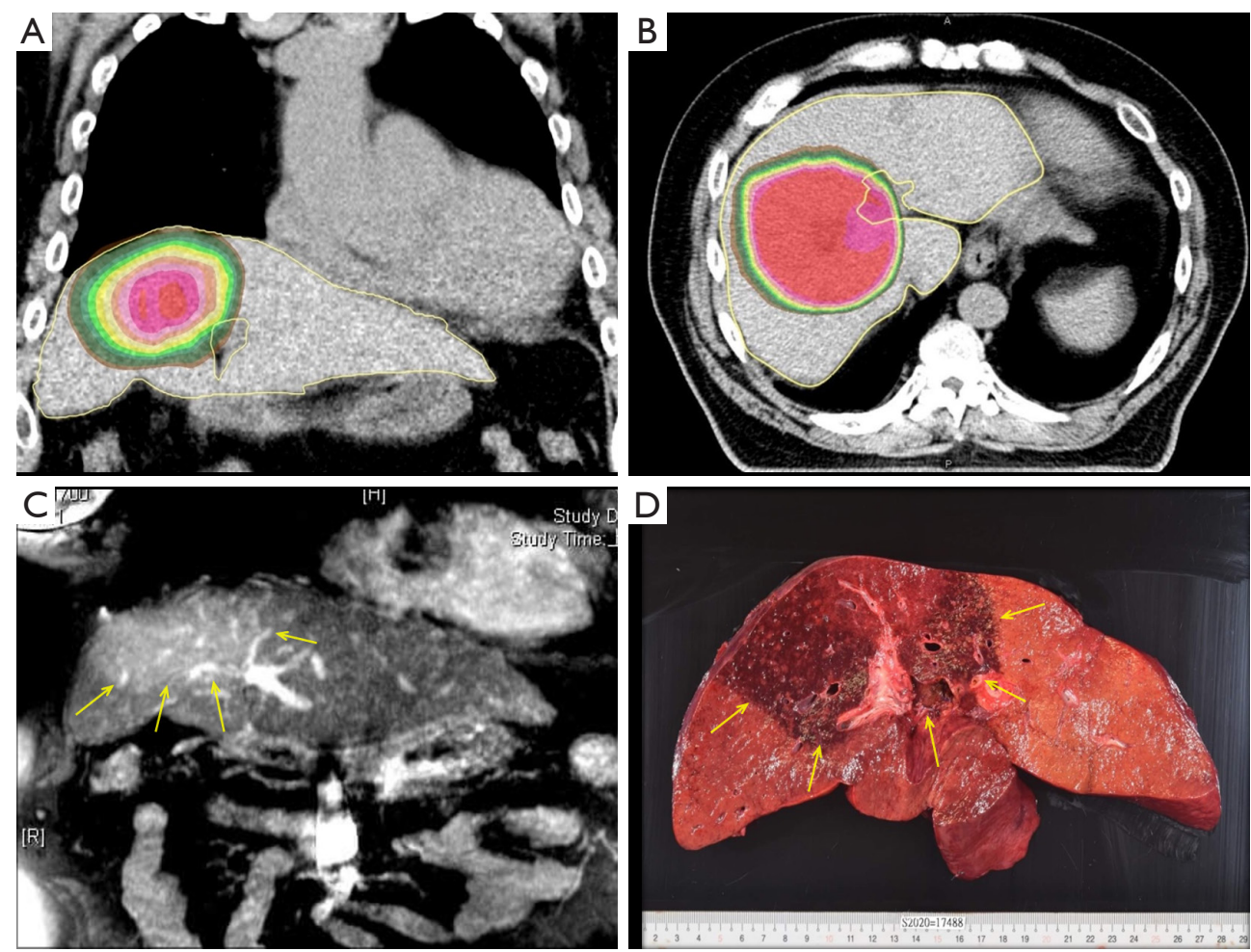

Figure 3 Proton beam therapy (PBT) of irradiated region (Case 1) shown by the color-washed area in the coronal view (A) and axial view (B). MRI (C) performed four weeks after completion of PBT shows clearly demarcated hyperintense area (arrows) coinciding with irradiated area. Coronal section of explant (D) showing gross hyperpigmented area with well-demarcated borders (arrows) coinciding with treated region and delineation of Bragg peaks.
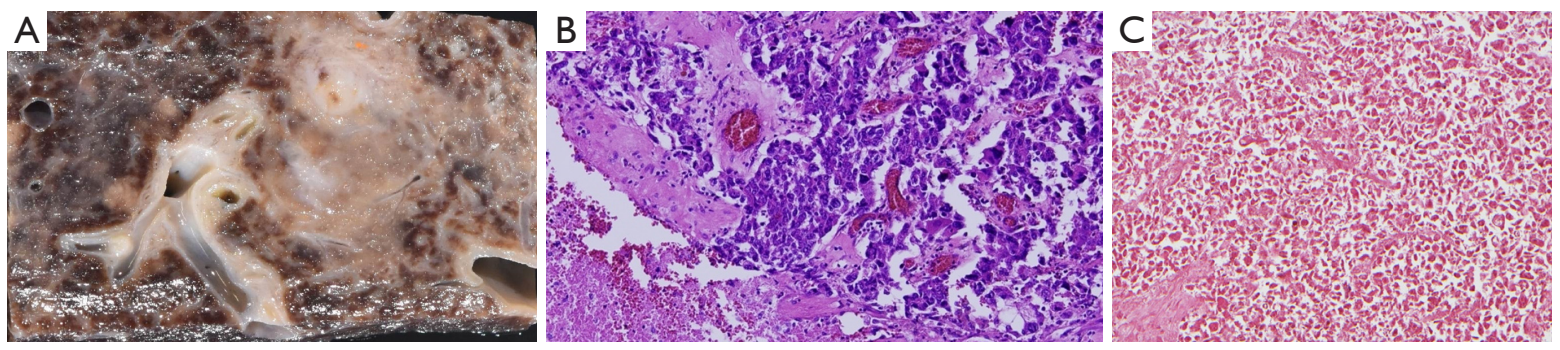

Figure 4 Gross explant pathology (Case 1) (A) shows residual S8 hepatocellular carcinoma (HCC) measuring $1.5 \mathrm{~cm}$. Final histopathology (B) shows a poorly differentiated HCC with at least $50 \%$ necrosis and presence of bile duct invasion [hematoxylin and eosin (H\&E) stain, $\times 66$ magnification]; HCC histopathology was consistent with an infiltrative scirrhous pattern. (C) Histopathology of distal common bile duct tissue shows complete necrosis of HCC (H\&E stain, $\times 66$ magnification).

with Infliximab for at least six years. She presented with a MELD of 20, CTP C12, with multiple HCC (AFP $3.5 \mathrm{ng} / \mathrm{dL}$ and CA19-9 $83.4 \mathrm{U} / \mathrm{mL}$ ) beyond the UCSF criteria (S1 $0.64 \mathrm{~cm}, \mathrm{~S} 83.8,1.2,0.63 \mathrm{~cm}, \mathrm{~S} 73.7 \mathrm{~cm}, \mathrm{~S} 6$ $2.4 \mathrm{~cm})$. PET scan revealed FDG-positivity of the S7 (SUVmax 9.7, TNR 3.7) and S8 (SUVmax 3.7, TNR
1.4) HCC foci (Figure $5 A, 5 B$ ). The patient however went into a decompensated state of liver cirrhosis (albumin $2.01 \mathrm{~g} / \mathrm{dL}$, bilirubin $7.3 \mathrm{mg} / \mathrm{dL}$, INR 1.73 , with hepatic encephalopathy, massive ascites) precluding any feasible locoregional treatment other than PBT. As with the first case, PBT was administered using the same protocol with a 

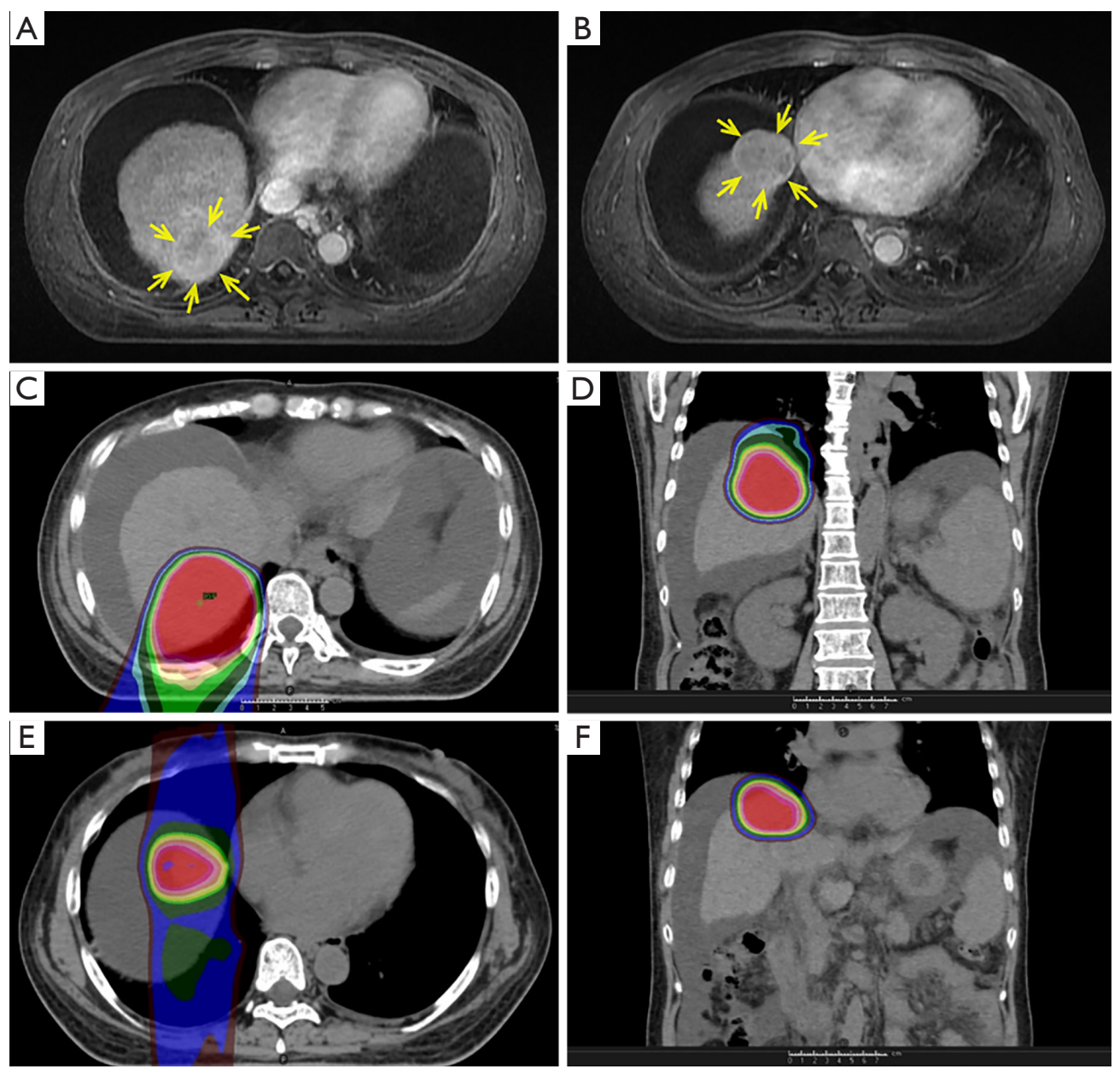

Figure 5 MRI (Case 2) axial views of the S7 $(3.7 \mathrm{~cm})(\mathrm{A})$ and S8 $(3.8 \mathrm{~cm})(\mathrm{B})$ hepatocellular carcinomas (HCCs) during initial presentation, both identified as FDG-positive on positron emission tomography (PET) scan. Axial (C,E) and coronal (D,F) views of the S7 and S8 HCCs respectively, with PBT-irradiated foci marked accordingly by the color-washed areas.

planned dose of $66 \mathrm{~Gy}$ (RBE) in 10 fractions for targeting the S7 and S8 FDG-positive lesions. However, an episode of cirrhosis-related esophageal variceal bleeding unrelated to PBT occurred during the treatment. The PBT was hence discontinued with a completed final dose of 52.8 Gy (RBE) administered over 8 fractions (Figure $5 C-5 F$ ). Reassessment $2 \frac{1}{2}$ weeks after treatment showed tumor downstaging, with almost complete metabolic response of the S7 HCC (Figure $6 A, 6 B$ ), and a decrease of the S8 SUVmax to 3.0, although TNR remained constant; although still out-ofcriteria, tumor biology and tumor burden improved, with an acceptable SUVmax and TNR, and one less HCC focus detected, respectively. After MDT discussion and consultation with the patient and her family regarding the 5 -year predicted HCC-specific survival with transplantation calculated at $82.7 \%$ based on the Metroticket 2.0 preoperative calculator, decision was made to proceed. The patient underwent LDLT with a right hepatic graft from her daughter six weeks after completion of PBT, with explant pathology revealing only five moderately differentiated residual viable tumors $[\mathrm{S} 73.8 \mathrm{~cm}$ (10\% necrosis), S8 $3.5 \mathrm{~cm}$ (5\% necrosis), 1.3 and $0.6 \mathrm{~cm}$ (100\% necrosis), S6 $2.0 \mathrm{~cm}, \mathrm{~S} 11.1 \mathrm{~cm}]$. The patient has also since been on dual immunosuppression therapy including a CNI and an mTor inhibitor, concurrently taken with lenvatinib, and has been recurrence-free ten months since transplantation.

\section{Discussion}

The new concept of transplant oncology has promulgated 

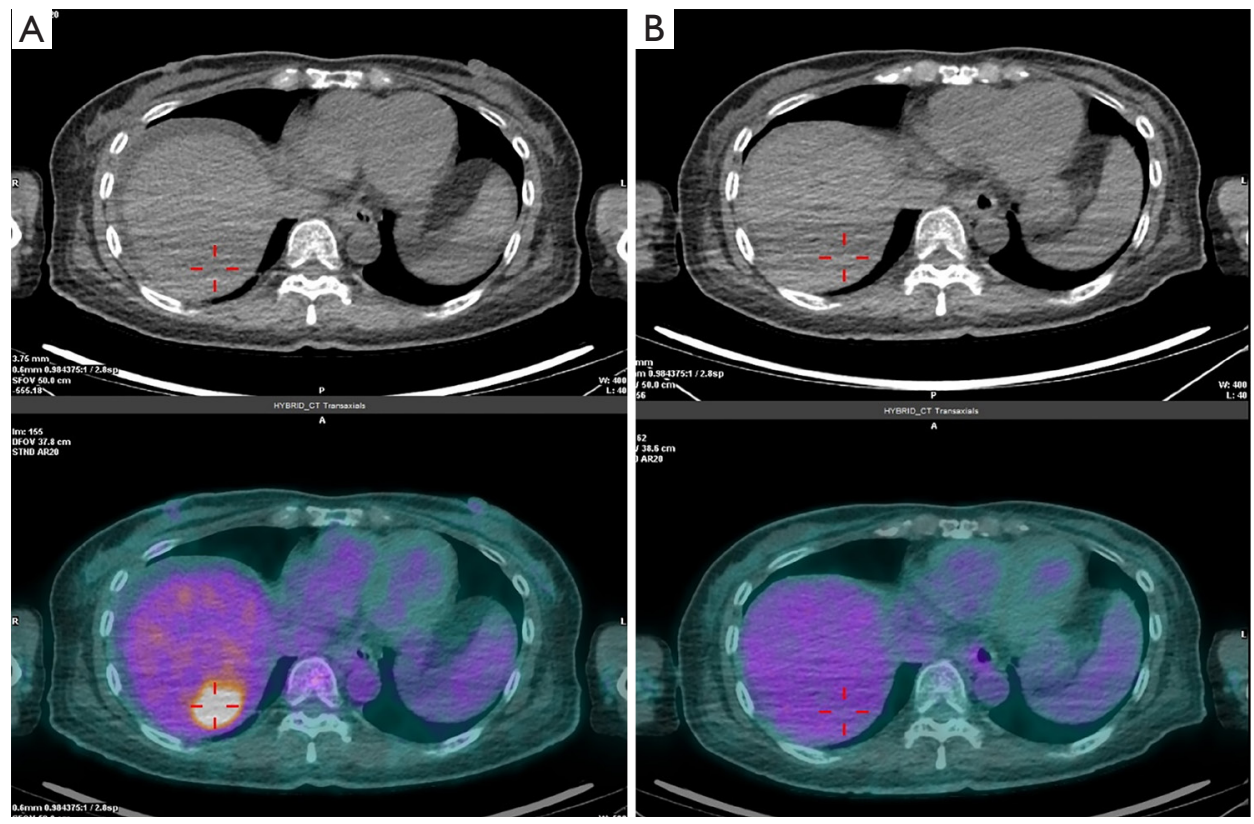

Figure 6 Positron emission tomography (PET) scan (Case 2) axial views of S7 hepatocellular carcinoma (HCC) showing tumor downstaging from measured SUVmax of 9.7, and TNR of 3.7 prior to proton beam therapy (PBT) (A), to almost complete metabolic response with no uptake two weeks after PBT (B).

the importance and relevance of understanding and addressing tumor biology on top of tumor burden in the management of HCC; an MDT of experts is necessary in order to optimize pre-LT therapy particularly of patients in the grey zone of LAHCC, to be able to offer the best survival outcomes and quality of life for HCC patients (7).

The current policy for HCC in KCGMH is to offer locoregional therapies in order to aggressively downstage LAHCC, while also extending recurrence-free and patient-specific survival by improving tumor biology and maximizing pathologic response prior to $\operatorname{LDLT}(8,9)$. We had previously published our findings on the prognosis of high FDG-avid HCC (SUVmax $>5$ and TNR >2) and found this group of patients as having a significantly poorer overall survival compared with recipients with negative or low FDG-avid patients $(10,11)$. Therefore, for HCCs with atypical imaging or are PET-positive with high avidity, we further obtain tissue-proof to exclude unfavorable pathologies, and likewise give consideration to bridging patients to transplantation. Understanding the potential of RT in improving prognostic outcomes in LAHCC, we therefore utilized PBT as locoregional therapy in the two cases presented.

In both cases, significant and treatment-altering clinical responses were observed within a few weeks of PBT.
Both patients exhibited improvements in tumor biology, while the second patient was furthermore downstaged in consideration of an elective LDLT. Both have thus far fared well postoperatively, although a longer observation period will still be needed.

Timing between PBT and LDLT is critical particularly for HCC patients with aggressive disease entities and/or impending liver failure. According to a Korean Study (12), it takes around six months for the full response of HCC to PBT to be observed, with a clinical in-field complete response rate of $69.2 \%$ according to the modified Response Evaluation Criteria in Solid Tumors (mRECIST) criteria. Learning from the relatively large experience in the use of neoadjuvant RT for locally advanced rectal cancer, further improvements in pathologic response from delayed surgery (up to eight weeks) does not portend any significant oncologic benefit compared to immediate surgery (within seven days after RT) $(13,14)$. Conclusively, LDLT was therefore performed within 4-6 weeks of PBT for the two presented cases after prognostic criteria was satisfied. The non-necrotic tumor was likely therefore due to the short waiting time to LDLT. Nonetheless, the precise clinical significance of greater pathologic response after the PBT is still unclear. While an optimal interval between PBT and LDLT remains unknown and is worth further investigation, 
an individualized approach in an era of precision oncology is at this time certainly the most prudent approach.

While the role of RT in the locoregional therapy of LAHCC becomes clearer, SBRT and other traditional photon modalities are still mainly limited by the allowable radiation dose due to the extent of adjacent organ injury. A proton beam has the physical advantage of creating a "Bragg peak" with a finite range of energy deposition with no exit dose beyond the target (Figure 3D). Therefore, compared with photon modalities, it is possible to solely irradiate a targeted liver tumor with higher doses using PBT while sparing surrounding tissues and organs (15), with consequently improved clinical outcomes $(16,17)$. Retrospective data have shown promising clinical results for PBT. The three-year local control rate ranges from $70 \%$ to $95 \%(18,19)$.

Compared with other known locoregional therapies including transarterial chemoembolization (TACE) and radiofrequency ablation (RFA), RT has the advantage of being non-invasive, painless, with very low biliary tract or vascular complication rates $(20,21)$. Conventional RT has already been demonstrated by the Toronto General Hospital group to be safe and efficacious in their initial cohort of 10 cases who were unsuitable for or failed bridging therapy to LT with TACE and/or RFA (22). It was also further demonstrated that stereotactic body RT (SBRT), while providing up to $68 \%$ pathologic response, was very well-tolerated, and could be safely utilized as an effective alternative to TACE or RFA for bridging therapy $(23,24)$. With vastly improved technological precision, PBT can safely deliver higher focused doses of radiation to tumors, enhancing its potential for positive tumor control and response (25).

Large liver tumors that cannot be optimally treated by TACE or RFA can be optimally irradiated by PBT (25). While transarterial radioembolization (TARE) with Yttrium-90 resins are also effective for PVTT, it has been limited to non-occluding PVTT, whereas higher grade PVTT up to first-order branches have seen promising response rates of $32.6-83 \%$ with RT according to various studies $(26,27)$. Therapeutically important, investigations have also shown that radiation not only improves the tumor biology and prognostic outcome, but rather also the immune microenvironment; this may have synergistic benefit when sequenced appropriately with other newer therapies such as immunotherapy (28).

Disadvantages still do exist with PBT. The paramount issue with this technology at present is availability as heavy investment is needed not only in terms of cost, but also space, limiting many institutions from building such a facility. Where available, applicability is still constrained by the physical property of protons being sensitive to organ movement, therefore making conformality and motion persistent challenges, albeit the improving techniques being developed to counter the problem (29).

Our cases demonstrate the potential of PBT as an important locoregional adjunct in LAHCC. It is critical nonetheless to recognize at this time that part of personalized precision care includes multidisciplinary considerations and the increasing role of RT in the form of PBT in bridging and downstaging selected high-risk patients for LDLT.

\section{Conclusion}

With the rapid pace of innovation in HCC diagnostics and therapeutics, the acceptance criteria for LDLT for HCC may now be further refined to be based not solely on morphology, but also on tumor biology. We have already studied how FDG-PET uptake highly correlates with HCC histopathology and has predictive value for recurrence $(10,11)$. PBT is a newer locoregional modality that shows great promise to be utilized for bridging aggressive HCC by improving tumor biology, or as a consideration for downstaging of LAHCC to within transplantation criteria, particularly where other locoregional treatments may be contraindicated. In today's rapidly progressing era of transplant and surgical oncology, beyond the mere fulfilment of national or institutional criteria for LT, the critical challenge for us to explore now would be how best to optimize survival outcomes of LT or surgery through an individualized yet realistic application of accessible locoregional and systemic therapies.

\section{Acknowledgments}

Funding: None.

\section{Footnote}

Provenance and Peer Review: This article was a standard submission to the journal. The article has undergone external peer review.

Conflicts of Interest: All authors have completed the ICMJE uniform disclosure form (available at https://hbsn.amegroups. 
com/article/view/10.21037/hbsn-21-379/coif). CLC serves as an unpaid editorial board member of Hepatobiliary Surgery and Nutrition. The other authors have no conflicts of interest to declare.

Ethical Statement: The authors are accountable for all aspects of the work in ensuring that questions related to the accuracy or integrity of any part of the work are appropriately investigated and resolved. Written informed consent was obtained from the patients for publication of this manuscript and any accompanying images.

Open Access Statement: This is an Open Access article distributed in accordance with the Creative Commons Attribution-NonCommercial-NoDerivs 4.0 International License (CC BY-NC-ND 4.0), which permits the noncommercial replication and distribution of the article with the strict proviso that no changes or edits are made and the original work is properly cited (including links to both the formal publication through the relevant DOI and the license). See: https://creativecommons.org/licenses/by-nc-nd/4.0/.

\section{References}

1. Barcena-Varela M, Lujambio A. The Endless Sources of Hepatocellular Carcinoma Heterogeneity. Cancers (Basel) 2021;13:2621.

2. Mazzaferro V, Sposito C, Zhou J, et al. Metroticket 2.0 Model for Analysis of Competing Risks of Death After Liver Transplantation for Hepatocellular Carcinoma. Gastroenterology 2018;154:128-39.

3. Rim CH, Kim CY, Yang DS, et al. Comparison of radiation therapy modalities for hepatocellular carcinoma with portal vein thrombosis: A meta-analysis and systematic review. Radiother Oncol 2018;129:112-22.

4. MOHW, Ministry of Health and Welfare. Information on the survival rate after organ transplantation in hospitals from April 1, 2005 to 2018 [Internet]. Taiwan; 2021 [cited 2021 Aug 24]. Available online: https://www.torsc.org.tw/ docDetail.jsp? uid=161\&pid=9\&doc_id=1358

5. Fukumitsu N, Sugahara S, Nakayama H, et al. A prospective study of hypofractionated proton beam therapy for patients with hepatocellular carcinoma. Int J Radiat Oncol Biol Phys 2009;74:831-6.

6. Mizumoto M, Okumura T, Hashimoto T, et al. Proton beam therapy for hepatocellular carcinoma: a comparison of three treatment protocols. Int J Radiat Oncol Biol Phys 2011;81:1039-45.
7. Hibi T, Sapisochin G. What is transplant oncology? Surgery 2019;165:281-5.

8. Concejero A, Chen CL, Wang CC, et al. Living donor liver transplantation for hepatocellular carcinoma: a single-center experience in Taiwan. Transplantation 2008;85:398-406.

9. Lin CC, Chen CL. Living donor liver transplantation for hepatocellular carcinoma achieves better outcomes. Hepatobiliary Surg Nutr 2016;5:415-21.

10. Hsu CC, Chen CL, Wang CC, et al. Combination of FDG-PET and UCSF Criteria for Predicting HCC Recurrence After Living Donor Liver Transplantation. Transplantation 2016;100:1925-32.

11. Ling LL, Hsu CC, Yong CC, et al. FDG-PET predicted unfavorable tumor histology in living donor liver transplant recipients; a retrospective cohort study. Int J Surg 2019;69:124-31.

12. Yu JI, Yoo GS, Cho S, et al. Initial clinical outcomes of proton beam radiotherapy for hepatocellular carcinoma. Radiat Oncol J 2018;36:25-34.

13. Bujko K, Nowacki MP, Nasierowska-Guttmejer A, et al. Long-term results of a randomized trial comparing preoperative short-course radiotherapy with preoperative conventionally fractionated chemoradiation for rectal cancer. Br J Surg 2006;93:1215-23.

14. Erlandsson J, Holm T, Pettersson D, et al. Optimal fractionation of preoperative radiotherapy and timing to surgery for rectal cancer (Stockholm III): a multicentre, randomised, non-blinded, phase 3, non-inferiority trial. Lancet Oncol 2017;18:336-46.

15. Wang X, Krishnan S, Zhang X, et al. Proton radiotherapy for liver tumors: dosimetric advantages over photon plans. Med Dosim 2008;33:259-67.

16. Sanford NN, Pursley J, Noe B, et al. Protons versus Photons for Unresectable Hepatocellular Carcinoma: Liver Decompensation and Overall Survival. Int J Radiat Oncol Biol Phys 2019;105:64-72.

17. Cheng JY, Liu CM, Wang YM, et al. Proton versus photon radiotherapy for primary hepatocellular carcinoma: a propensity-matched analysis. Radiat Oncol 2020;15:159.

18. Yoo GS, Yu JI, Park HC. Proton therapy for hepatocellular carcinoma: Current knowledges and future perspectives. World J Gastroenterol 2018;24:3090-100.

19. Chadha AS, Gunther JR, Hsieh CE, et al. Proton beam therapy outcomes for localized unresectable hepatocellular carcinoma. Radiother Oncol 2019;133:54-61.

20. Yu JI, Park HC, Lim DH, et al. Do Biliary Complications after Hypofractionated Radiation Therapy in 
Hepatocellular Carcinoma Matter? Cancer Res Treat 2016;48:574-82.

21. Yoo GS, Yu JI, Park HC, et al. Do Biliary Complications after Proton Beam Therapy for Perihilar Hepatocellular Carcinoma Matter? Cancers (Basel) 2020;12:2395.

22. Sandroussi C, Dawson LA, Lee M, et al. Radiotherapy as a bridge to liver transplantation for hepatocellular carcinoma. Transpl Int 2010;23:299-306.

23. Mannina EM, Cardenes HR, Lasley FD, et al. Role of Stereotactic Body Radiation Therapy Before Orthotopic Liver Transplantation: Retrospective Evaluation of Pathologic Response and Outcomes. Int J Radiat Oncol Biol Phys 2017;97:931-8.

24. Sapisochin G, Barry A, Doherty M, et al. Stereotactic body radiotherapy vs. TACE or RFA as a bridge to transplant in patients with hepatocellular carcinoma. An intention-totreat analysis. J Hepatol 2017;67:92-9.

25. Sugahara S, Oshiro Y, Nakayama H, et al. Proton beam

Cite this article as: Chen CL, Ong AD, Cheng JY, Yong CC, Lin CC, Chen CY, Cheng YF. Proton beam therapy to bridge or downstage locally advanced hepatocellular carcinoma to living donor liver transplantation. HepatoBiliary Surg Nutr 2022;11(1):103-111. doi: 10.21037/hbsn-21-379 therapy for large hepatocellular carcinoma. Int J Radiat Oncol Biol Phys 2010;76:460-6.

26. Somma F, Stoia V, Serra N, et al. Yttrium-90 transarterial radioembolization in advanced-stage HCC: The impact of portal vein thrombosis on survival. PLoS One 2019;14:e0216935.

27. Cerrito L, Annicchiarico BE, Iezzi R, et al. Treatment of hepatocellular carcinoma in patients with portal vein tumor thrombosis: Beyond the known frontiers. World J Gastroenterol 2019;25:4360-82.

28. Lee YH, Tai D, Yip C, et al. Combinational Immunotherapy for Hepatocellular Carcinoma:

Radiotherapy, Immune Checkpoint Blockade and Beyond. Front Immunol 2020;11:568759.

29. Eggert T, Greten TF. Current Standard and Future Perspectives in Non-Surgical Therapy for Hepatocellular Carcinoma. Digestion 2017;96:1-4. 